\title{
Risk factors predicting upper urinary tract deterioration in patients with spinal cord injury: a prospective study
}

\author{
Z Zhang ${ }^{1,2,3,4}$ and L Liao ${ }^{1,2,4}$
}

\begin{abstract}
Study design: A prospective study.
Objectives: To investigate the risk factors predicting upper urinary tract (UUT) deterioration in patients with spinal cord injury (SCI). Setting: Beijing, China.

Methods: We reviewed the medical records and UUT imaging studies of 112 patients, with a mean follow-up of 2 years. Variables evaluated for possible influence on UUTs included patient age, gender and educational background; injury level and completeness; bladder management method; and the presence of adverse outcomes such as recurrent urinary tract infections and bladder stones. Video urodynamic data were also reviewed. Total renal function was assessed by serum creatinine. UUT abnormalities were assessed by renal ultrasound or magnetic resonance imaging.

Results: Lumbosacral spinal cord lesions likely contribute to UUT deterioration. UUT abnormalities were present in 23 patients $(65.7 \%)$ in a spontaneous voiding group, 10 patients $(20 \%)$ in a clean intermittent catheterization group, 15 patients $(78.9 \%)$ in an indwelling urethral catheterization group and 7 patients $(87.5 \%)$ in a suprapubic Foley catheterization group $(P<0.001)$. When dividing bladder management method into two groups, catheter-free (spontaneous and intermittent voiding) and indwelling catheter (urethral and suprapubic catheterization), there was UUT dysfunction in 33 patients (38.3\%) and 22 patients (81.5\%), respectively $(P<0.001)$.
\end{abstract}

Conclusions: Lumbosacral $\mathrm{SCl}$ and chronic indwelling urethral and suprapubic catheterization were predictors of UUT deterioration. Spinal Cord (2014) 52, 468-471; doi:10.1038/sc.2014.63; published online 29 April 2014

\section{INTRODUCTION}

Neurogenic bladder is one of the bladder and/or urethral dysfunctions caused by nervous system disease, and spinal cord injury (SCI) is one of the most common causes. In the 1950 s, $45-50 \%$ of patients with SCI died of renal failure and uremia secondary to SCI. ${ }^{1}$ Neurogenic bladder management methods have changed enormously in the last 60 years, especially with the development of neurology and introduction of sterile or clean intermittent catheterization (IC); the morbidity of upper urinary tract (UUT) lesion has decreased to $3-5 \%$, and the mortality caused by renal failure also decreased significantly. Nevertheless, many current studies indicate that urinary complications are still the main causes of death during the later stage in SCI. A recent large-scale epidemiological study in the United States showed that $29-36 \%$ of patients with SCI suffer from lower urinary tract infection. ${ }^{2}$ Several factors associated with UUT deterioration in patients with SCI need further study: the risk factors contributing to UUT in patients with SCI should be identified early to allow for proper clinical intervention, protect UUT function, and improve patients' quality of life after injury.

\section{MATERIALS AND METHODS}

The Ethical Committee at China Rehabilitation Research Center approved the study. In this prospective study, 150 patients with SCI were recruited from March 2009 to March 2011. Inclusion criteria included the following:
(1) initial diagnosis of SCI (complete or incomplete), with the diagnosis conforming to International Standards for Neurological Classification of SCI revised in 2002 by the American Spinal Injury Association (ASIA), and confirmed by spinal CT and/or MRI; (2) after spinal shock recovery clinically; (3) patients were aged 18-60 years; (4) normal UUT; (5) without therapies of anticholinergic agents and Botulinum toxin injection. Exclusive criteria: patients with upper urinary deterioration. Most of the patients received urodynamic test after the end of spinal shock, but 38 patients with SCI were excluded from the study due to the lack of initial urodynamic data.

For the remaining 112 consecutive patients, observation data included the following: (1) general demographics (age, gender, education background); (2) SCI characteristics (neurologic level of injury and injury completeness); (3) bladder management methods (spontaneous voiding, IC, transurethral indwelling catheterization or suprapubic catheterization); (4) urinary complications (infection and calculus): the urinary tract infection was defined as the bacteriuria count $\geqslant 10^{5} \mathrm{ml}^{-1}$ conjoint with leukocyturia $\geqslant 100 \mathrm{~mm}^{-3}$, or with the symptoms including chills, fever, sweating, cloudy/malodorous urine, et al.; and (5) urodynamic (VUD) parameters (maximum cystometric capacity (MCC), bladder compliance (BC), detrusor overactivity, residual urine volume), maximum urethral closure pressure, detrusor leak point pressure (DLPP), detrusor sphincter dyssynergia (DSD) and maximum detrusor pressure).

The UUT was considered as deterioration if any of the following were described unilaterally or bilaterally: (1) any grade of vesicoureteral reflux assessed by VUD examination; $(2) \geqslant 1$ grade of hydronephrosis according to the Society for Fetal Urology ultrasound grading system, ${ }^{3}(3) \geqslant 1$ grade of

${ }^{1}$ Department of Urology, China Rehabilitation Research Center, Beijing, China; ${ }^{2}$ School of Rehabilitation Medicine, Capital Medical University, Beijing, China and ${ }^{3}$ Department of Rehabilitation Medicine, The Second Affiliated Hospital of Zhejiang University School of Medicine, Hangzhou, China

${ }^{4}$ Zhihua Zhang and Limin Liao are considered co-first authors.

Correspondence: Professor L Liao, Department of Urology, China Rehabilitation Research Center, No 10 Jiaomen Beilu, Beijing 100068, China.

E-mail: Imliao@263.net

Received 9 January 2014; revised 26 March 2014; accepted 1 April 2014; published online 29 April 2014 
UUT dilation according to Liao's magnetic resonance urography grading system: ${ }^{4}$ Grade 1: there is slight separation of the central renal complex, but no visualized calices; grade 2: renal pelvis is dilated, calices may be visualized, but renal parenchyma is normal; grade 3: renal pelvis is further dilated and there are fluid-filled calices throughout the kidney, and renal parenchyma is beginning to thin; grade 4 : as in grade 3 , but the renal parenchyma is significantly thinned. (4) elevated serum creatinine $\left(>133 \mu \mathrm{moll}^{-1}\right)$. The hydronephrosis were evaluated by ultrasound and serum creatinine level was examined every 6 months, and the VUD was performed every one year. The end point of study was defined as hydronephrosis or UUT dilation or vesicoureteral reflux occurring; however, the isotope renography was performed if the UUT deterioration was confirmed. The longest fellow-up period was 3 years.

Statistical analysis of the data was performed using SPSS 11.5.0 (SPSS, Chicago, IL, USA). Descriptive statistics were reported as the mean \pm s.d. The $\chi^{2}$-test or one-way analysis of variance was used to assess the associations between the various potential risk factors and UUT. We performed COX regression analysis of UUT deterioration versus general demographic data characteristics, SCI characteristics, bladder management methods, urinary complications and urodynamic parameters. Statistical significance was set at a $P<0.05$.

\section{RESULTS}

All patients were followed up for mean 24 months (range from 6 to 36 months). The 112 patients ranged in age from 18 to 57 years, with 91 males and 21 females. Serum creatinine values were observed to be normal for each patient before and after follow-up. In total, 55 patients $(49.1 \%)$ had UUT deterioration at the end (48 males and 7 females).

Table 1 shows that patient's with characteristics of $>45$ years of age, or male, or < high school education had a slightly higher incidence of UUT deterioration: $59.1 \%, 52.7 \%$ and $55 \%$, respectively, but these differences were not statistically significant $(P>0.05)$.

Of the 112 patients, 30.3, 45.5 and $24.1 \%$ had cervical, thoracic and lumbosacral spinal cord injuries, respectively; and the frequency of UUT deterioration in these groups was $35.3 \%, 51.0 \%, 63 \%$, respectively. There were significant differences between cervical and lumbosacral level groups $(P=0.032)$. Table 1 also shows the association between UUT and completeness of injury $(P=0.888)$, which was not statistically significant.

The most common bladder management method was IC, followed by spontaneous voiding, indwelling urethral catheterization and suprapubic catheterization. Table 1 shows the incidence of UUT deterioration in patients with spontaneous voiding, IC, indwelling urethral catheterization and suprapubic catheterization was $65.7 \%$, $20 \%, 78.9 \%$ and $87.5 \%$, respectively. We noted significant difference among bladder management methods $(P<0.001)$.

According to the duration of catheter contact with lower urinary tract, bladder management methods were divided into a catheter-free (spontaneous voiding and IC) and an indwelling catheter (indwelling urethral catheterization and suprapubic catheterization) group. We found that normal UUT were more common in catheter-free patients (Table 1). The incidence of deterioration in the indwelling catheter group was $81.5 \%$, which was much higher than that in catheter-free group $(38.3 \%)$, indicating that an indwelling catheter is highly significantly correlated with UUT deterioration $(P<0.001)$.

Repeated lower urinary tract infection and bladder calculus are the most common complications in patients with post-traumatic SCI. Our investigation found no statistically significant difference in rates of infection or calculus associated with UUT deterioration (Table 1).

Table 2 shows that objective urodynamic assessment of the lower urinary tract is significantly correlated with UUT in patients with SCI.
Table 1 The relationship of demographic characteristics, SCI characteristics, bladder management methods, catheter and urinary complications with upper urinary tract in 112 patients with $\mathrm{SCl}$

Parameters No. of patients \begin{tabular}{c} 
Upper urinary tract \\
\cline { 2 - 4 }
\end{tabular}

\begin{tabular}{|c|c|c|c|c|}
\hline \multicolumn{5}{|c|}{ Demographic characteristics } \\
\hline \multicolumn{5}{|c|}{ Age } \\
\hline$<45$ & 90 & $42(46.7)$ & $48(53.3)$ & \\
\hline$\geqslant 45$ & 22 & $13(59.1)$ & $9(40.9)$ & 0.296 \\
\hline \multicolumn{5}{|l|}{ Gender } \\
\hline Male & 91 & $48(52.7)$ & $43(47.3)$ & \\
\hline Female & 21 & $7(33.3)$ & $14(66.7)$ & 0.109 \\
\hline \multicolumn{5}{|c|}{ Education background } \\
\hline$<$ High school & 60 & $33(55.0)$ & $27(45.0)$ & \\
\hline$>$ High school & 52 & $22(42.3)$ & $30(57.7)$ & 0.180 \\
\hline \multicolumn{5}{|l|}{ SCl characteristics } \\
\hline \multicolumn{5}{|l|}{ Injury type } \\
\hline Cervical & 34 & $12(35.3)$ & $22(64.7)$ & 0.154 \\
\hline Thoracic & 51 & $26(51.0)$ & 25 (49.0) & 0.311 \\
\hline Lumbosacral & 27 & $17(63.0)$ & $10(37.0)$ & $0.032^{*}$ \\
\hline
\end{tabular}

\section{Injury completeness}

$\begin{array}{llll}\text { Complete A } & 72 & 35(48.6) & 37(51.4)\end{array}$

Incomplete B-E $\quad 40 \quad 20(50.0) \quad 20(50.0)$

0.888

Bladder management methods

Indwelling urethral catheterization

$\begin{array}{lllll}\text { Yes } & 19 & 15(78.9) & 4(21.1) & 0.000 *\end{array}$

Suprapubic catheterization

Yes 8

$7(87.5) \quad 1(12.5)$

0.309

Intermittent catheterization

Yes 50

$10(20.0)$

$40(80.0)$

$0.000 *$

Spontaneous voiding

Yes

35

$23(65.7)$

12 (34.3)

0.000 *

Catheter

Catheter-free

Yes

85

$33(38.8)$

$52(61.2)$

Indwelling catheter

Yes

27

$22(81.5)$

5 (18.5)

0.000 *

Urinary complications

Lower urinary tract infection

Yes 91

No

21

$48(52.7)$

$43(47.3)$

$7(33.3) \quad 14(66.7)$

0.109

Bladder calculus

$\begin{array}{llll}\text { Yes } & 17 & 7(41.2) & 10(58.8)\end{array}$

No $\quad 95 \quad 48(50.5) \quad 47(49.5)$

0.478

Abbreviation: $\mathrm{SCl}$, spinal cord injury.

*Statistically significant. 
Table 2 The relationship of urodynamic parameters with upper urinary tract in 112 patients with $\mathrm{SCl}$

\begin{tabular}{|c|c|c|c|c|}
\hline \multirow[t]{2}{*}{ Parameters } & \multirow[t]{2}{*}{ No. of patients } & \multicolumn{2}{|c|}{ Upper urinary tracts } & \multirow[t]{2}{*}{ P-value } \\
\hline & & Abnormal no. (\%) & Normal no. (\%) & \\
\hline \multicolumn{5}{|l|}{$\operatorname{MCC}(\mathrm{ml})$} \\
\hline$<200$ & 26 & $18(69.2)$ & $8(30.8)$ & \\
\hline$\geqslant 200$ & 86 & $37(43.0)$ & $49(57.0)$ & $0.019 *$ \\
\hline \multicolumn{5}{|c|}{ Pdet.max $\left(\mathrm{cm} \mathrm{H}_{2} \mathrm{O}\right)$} \\
\hline$<40$ & 38 & $14(36.8)$ & $24(63.2)$ & \\
\hline$\geqslant 40$ & 74 & $41(55.4)$ & $33(44.6)$ & 0.063 \\
\hline \multicolumn{5}{|c|}{$\mathrm{BC}\left(\mathrm{m} / \mathrm{cm} \mathrm{H} \mathrm{H}_{2} \mathrm{O}^{-1}\right)$} \\
\hline$<20$ & 87 & $48(55.2)$ & $39(44.8)$ & \\
\hline$\geqslant 20$ & 23 & $5(21.7)$ & $18(78.3)$ & $0.004 *$ \\
\hline \multicolumn{5}{|l|}{$D S D$} \\
\hline Yes & 51 & $30(58.8)$ & $21(41.2)$ & \\
\hline No & 61 & 25 (41.0) & $36(59.0)$ & 0.060 \\
\hline
\end{tabular}

Abbreviations: BC, bladder compliance; DSD, detrusor sphincter dyssynergia; MCC, maximum cystometric capacity; Pdet.max, maximum detrusor pressure; $\mathrm{SCl}$, spinal cord injury.

*Statistically significant

In the MCC $<200 \mathrm{ml}$ group, $69.2 \%$ of patients had UUT deterioration and in the MCC $\geqslant 200 \mathrm{ml}$ group, the rate was $43.0 \%(P=0.019)$. We noted a significantly higher incidence of UUT deterioration in patients with $\mathrm{MCC}<200 \mathrm{ml}$ than $\mathrm{MCC} \geqslant 200 \mathrm{ml}$. As to $\mathrm{BC}$, the frequency of UUT deterioration in $\mathrm{BC}<20 \mathrm{ml} \mathrm{cm}^{-1}$ water and BC $\geqslant 20 \mathrm{ml} \mathrm{cm}^{-1}$ water group was 55.2 and $21.7 \%$, respectively, UUT deterioration was significantly more common in those with BC $<20 \mathrm{ml} \mathrm{cm}^{-1}$ water versus $\mathrm{BC} \geqslant 20 \mathrm{ml} \mathrm{cm}^{-1}$ water $(P=0.004)$. Univariate analysis noted that the rate of UUT deterioration in maximum detrusor pressure $\geqslant 40 \mathrm{~cm}$ water at filling stage was $55.4 \%$ and maximum detrusor pressure $<40 \mathrm{~cm}$ water was $36.8 \%$ $(P=0.063)$. In patients with DSD, the frequency was $58.8 \%$, whereas without DSD it was $41.0 \%(P=0.06)$. Our findings strongly suggest that patients with DSD and/or maximum detrusor pressure $\geqslant 40 \mathrm{~cm}$ water have a greater prevalence of UUT deterioration, although both of them approached significance $(P=0.06)$. If there were a larger sample size, the result might be different.

Multiple COX regression analysis of UUT deterioration in relation to general demographic information, SCI characteristics, bladder management methods, urinary complications and urodynamic parameters reveals that injury level and bladder management method were significantly associated with UUT abnormalities. The relative risk of upper urinary deterioration in lumbosacral SCI was 4.204, and indwelling urethral catheterization was 5.819, which is the most important risk factor.

\section{DISCUSSION}

Neurogenic bladder and urethral dysfunction secondary to SCI is a direct cause of UUT deterioration. In addition, treatment methods, bladder management methods, autonomic nervous disorder, recurrent lower urinary infection, bladder calculus might have important roles. Sekar et al. ${ }^{5}$ found that renal functioning was more likely to be compromised among women compared with men in the long term after injury, and that aging may be another risk factor. Weld et al. ${ }^{6}$ also noted that elderly patients with SCI were more likely to incur UUT damage. This may be because they tended to prefer indwelling catheterization, and/or due to age-based differences in their renal function before injury. After these two factors had been ruled out, age seemed to have nothing to do with renal function. Consistent with these studies, we did not find a significant association between age and gender and UUT post-traumatic SCI. As different educational backgrounds might produce various understandings and treatment attitudes toward neurogenic bladder, we examined this factor in our study. The results noted that patients who had not completed high school might be more likely to suffer from upper tract deterioration, although without statistical significance. Sekar et al..$^{5}$ found the effective renal plasma flow in patients with cervical SCI was commonly lower compared with other levels of injury. PetterssonHammerstad et al. ${ }^{7}$ stated that in the early stages of SCI, the glomerular filtration rate in all patients decreased to $86 \%$ of reference value. Level and completeness of SCI (ASIA A) may have an important role in renal dysfunction. Our findings revealed that in the long term, renal functioning was more likely to be compromised among patients with lumbosacral SCI versus cervical and thoracic SCI; although their serum creatinine values were normal all the time since investigation, the imaging of UUT deterioration predicted that the kidneys probably were at the stage of functional compensation. Meanwhile multiple COX regression analysis indicated that lumbosacral level SCI was one of the important risk factors predicting UUT lesion. Weld et al. ${ }^{8}$ reported that low BC and high DLPP may be associated with sacral SCI. Our findings are consistent with these findings. We presumed that patients with lumbosacral SCI may have a higher incidence of low BC. The pathogenesis of UUT dilation or hydronephrosis associated with low BC may be caused by ureteral stricture obstruction of wall segment because of detrusor fibrosis and bladder wall thickening, which has been confirmed by magnetic resonance urography and isotope nephrogram. In our investigation, completeness of injury appeared to be irrelevant to UUT function. Alternatively serum creatinine was selected to assess renal function but no abnormal values were found. Thus, our diagnostic criteria for UUT deterioration mainly depend on ultrasound and/or MRI. However, ultrasound and/or MRI were not comparable with glomerular filtration rate or effective renal plasma flow, so we still cannot completely clarify the relationship between the completeness of SCI and renal function.

UUT deterioration seriously impact long-term lives in patients with SCI. With clean IC currently being widely considered the safest bladder management method, VUD examination generally has been accepted as a means of identifying neurogenic urinary tract dysfunction and assessing further UUT abnormalities. Previous investigators have suggested that high DLPP, low BC and DSD are significantly associated with UUT deterioration. UUT were more likely to be compromised among patients with DLPP $>40 \mathrm{~cm}$ water. The low BC, detrusor overactivity and DLPP $>40 \mathrm{~cm}$ water were related to UUT lesions, and were the main risk factors. ${ }^{9}$ Chronic high bladder pressure during the filling stage seems to highly predict the potential UUT dilatation. Our VUD evaluation indicated that BC $<20 \mathrm{ml} \mathrm{cm}^{-1}$ water and/or MCC $<200 \mathrm{ml}$ was significantly associated with the incidence of UUT deterioration. Low BC and decreased capacity may lead to high bladder pressure during the filling phase, which may gradually aggravate the ureteral workload carrying urine down to the bladder, and damage the vesicoureteral antireflux mechanism. The ureteral function decompensated with time and vesicoureteral reflux, and then ureteral dilation and hydronephrosis developed. Clinical practices on neurogenic bladder have confirmed that if bladder capacity enlarges and compliance improves, the whole bladder filling phase could be sustained at 
sufficient volume and low pressure, which appears, to some extent, to alleviate and even prevent UUT impairment.

The optimal bladder management method for patients with SCI still remains controversial. Various factors should be considered in lower urinary tract management, including comfort and convenience, renal protection, urinary tract complication prevention, and so on. Balance, regularity, low bladder pressure, timely evacuation, no residual or leakage of urine were the goals of patients suffering from long-term neurogenic bladder. Clean IC has been thought of as the gold standard for these patients. ${ }^{6,10}$

Hackler et al. ${ }^{11}$ noted that the renal impairment after suprapubic catheterization for 5 years was similar to that of indwelling catheterization after 20 years. In other words, the damage of suprapubic catheterization to the UUT was more severe than that of indwelling urethral catheterization. Chao et al. ${ }^{12}$ did not find a significant difference in creatinine clearance between indwelling urethral catheterization and spontaneous voiding. However, the frequency of renal scarring and UUT dilation in the former was higher than the latter. Bennett et al. ${ }^{13}$ stated that indwelling urethral catheterization may aggravate urinary complications and increase the incidence of UUT impairment. Larsen et al. ${ }^{14}$ have demonstrated that patients with chronic indwelling catheterization were more likely to suffer from progressive UUT damage. Conversely, Weld et al. ${ }^{6}$ emphasized that all methods of bladder management were beneficial for UUT protection. Nevertheless, proteinuria and reduced creatinine clearance in the indwelling urethral catheterization group were independent risk signals for renal impairment. $\mathrm{Ku}$ et $a l .{ }^{15}$ found that the incidence of UUT deterioration in patients with post-traumatic SCI was $32.4 \%$, especially indwelling urethral catheterization group; they concluded that indwelling urethral catheterization was an independent risk factor. However, contrary to Hackler et al., ${ }^{11}$ the results also demonstrated that damage to renal function from suprapubic catheterization seemed to be milder than indwelling urethral catheterization. Alternatively, some investigators observed that renal function altered slightly after traumatic injury and remained nearly the same as before injury, regardless of the bladder management method used. ${ }^{7}$ In our study, we divided the bladder management methods into spontaneous voiding, IC, indwelling urethral catheterization and suprapubic catheterization groups. Univariate analysis showed that there were significant differences between the four groups in UUT deterioration. Multiple COX regression analysis indicated that indwelling urethral catheterization is the most important risk factor for UUT deterioration. Chronic indwelling catheterization may induce inflammation of the lower urinary tract and even lead to repeated infections. Lower urinary tract inflammation, infection and constant bladder decompression may accelerate bladder wall fibrosis. Larsen suggested that severe detrusor fibrosis histologically was likely a risk factor in UUT deterioration. ${ }^{16}$ Sekar et al. ${ }^{5}$ noted that reduced renal function seems to be related to renal calculus. Our study also indicates that the relationship between bladder calculus and UUT still seems to be unclear.

\section{CONCLUSIONS}

Our investigation showed that chronic indwelling urethral and suprapubic catheterization and lumbosacral level of SCI were two crucial risk factors for UUT deterioration. Urodynamic parameters with high detrusor pressure also were dangerous signals of UUT deterioration.

\section{DATA ARCHIVING}

There were no data to deposit.

\section{CONFLICT OF INTEREST}

The authors declare no conflict of interest.

\section{ACKNOWLEDGEMENTS}

This study was supported by China National Technology R\&G Program (2012BAI34B02).

1 Whiteneck GG, Charlifue SW, Frankel HL, Fraser MH, Gardner BP, Gerhart KA et al. Mortality, morbidity, and psychosocial outcomes of persons spinal cord injured more than 20 years ago. Paraplegia 1992; 30: 617

2 Manack A, Motsko SP, Haag-Molkenteller C, Dmochowski RR, Goehring EL Jr, Nguyen-Khoa BA et al. Epidemiology and healthcare utilization of neurogenic bladder patients in a US claims database. Neurourol Urodyn 2011; 30: 395-401.

3 Fernbach SK, Maizels M, Conway JJ. Ultrasound grading of hydronephrosis: introduction to the system used by the Society for Fetal Urology. Pediatr Radiol 1993; 23: 478-480.

4 Liao LM, Zhang F. Upper urinary tract dilation in the neurogenic bladder. In: Santucci RA and Chen M (eds) Ureters: Anatomy, Physiology and Disorders. Nova Science Publishers: New York, 2012, pp 99-110.

5 Sekar P, Wallace DD, Waites KB, DeVivo MJ, Lloyd LK, Stover SL et al Comparison of long-term renal function after spinal cord injury using different urinary management methods. Arch Phys Med Rehabil 1997; 78: 992-997.

6 Weld KJ, Wall BM, Mangold TA, Steere EL, Dmochowski RR. Influences on renal function in chronic spinal cord injured patients. J Urol 2000; 164: 1490-1493.

7 Pettersson-Hammerstad K, Jonsson O, Svennung IB, Karlsson AK. Impaired renal function in newly spinal cord injured patients improves in the chronic state-effect of clean intermittent catheterization? J Urol 2008; 180: 187-191.

8 Weld KJ, Dmochowski RR. Association of level of injury and bladder behavior in patients with post-traumatic spinal cord injury. Urology 2000; 55: 490-494.

9 Gerridzen RG, Thijssen AM, Dehoux E. Risk factors for upper tract deterioration in chronic spinal cord injury patients. J Urol 1992; 147: 416-418.

10 Zommick JN, Simoneau AR, Skinner DG, Ginsberg DA. Continent lower urinary tract reconstruction in the cervical spinal cord injured population. J Urol 2003; 169: 2184-2187.

11 Hackler RH. Long-term suprapubic cystostomy drainage in spinal cord injury patients. Br J Urol 1982; 54: 120-121.

12 Chao R, Clowers D, Mayo ME. Fate of upper urinary tracts in patients with indwelling catheters after spinal cord injury. Urology 1993; 42: 259-262.

13 Bennett CJ, Young MN, Adkins RH, Diaz F. Comparison of bladder management complication outcomes in female spinal cord injury patients. J Urol 1995; 153: 1458-1460.

14 Larsen LD, Chamberlin DA, Khonsari F. Retrospective analysis of urologic complications in male patients with spinal cord injury managed with and without indwelling urinary catheters. Urology 1997; 50: 418-422.

$15 \mathrm{Ku}$ JH, Choi WJ, Lee KY, Jung TY, Lee JK, Park WH et al. Complications of the upper urinary tract in patients with spinal cord injury: a long-term follow-up study. Urol Res 2005; 33: 435-439.

16 Ozkan B, Demirkesen O, Durak H, Uygun N, Ismailoglu V, Cetinel B. Which factors predict upper urinary tract deterioration in overactive neurogenic bladder dysfunction? Urology 2005; 66: 99-104. 